\title{
Présence au Nord-Tchad de Eupolystoma alluaudi (de Beauchamp, 1913) (Monogenea, Polystomatidae)
}

\author{
Par Louis EUZET et Claude COMBES
}

En 1913, P.-M. de Beauchamp décrit, sous le nom de Polystomum alluaudi un Monogène récolté par la Mission Alluaud et Jeannel en Afrique Orientale. L'exemplaire unique de ce parasite avait été trouvé au cours du triage des animaux capturés dans une mare temporaire et provenait donc vraisemblablement de l'un des Amphibiens présents dans cette mare, à savoir Bufo regularis Reuss et Bufo taitanus Peters.

En 1935, Ozaki crée le genre Parapolystoma avec comme type P. bulliense (Johnston, 1912). Ce genre se caractérise principalement par: un testicule apparemment simple situé dans la partie médiane du corps ; un utérus long s'étendant dans la partie postérieure du corps (donc en arrière du testicule) ; un tube digestif sans anastomose haptoriale ni transverse ; un hapteur pourvu d'une paire de grands crochets (hamulis).

Ozaki propose de placer l'espèce de de Beauchamp dans ce nouveau genre. Mais aucun des caractères précités ne se retrouve ni dans la description, ni dans le dessin concernant Polystomum alluaudi et nous avons pu vérifier cette incompatibilité sur le matériel original de P.-M. de Beauchamp. La proposition d'Ozaki est donc indéfendable.

En 1962, M. Beverley-Burton pense retrouver chez Bufo regularis Reuss et Rana adspersa (Tschudi) l'espèce de de Beauchamp et lui conserve le statut de Parapolystoma alluaudi (de Beauchamp, 1913) Ozaki, 1935.

En 1963, Yamaguti, prenant conscience des incompatibilités que nous venons de souligner, crée le genre Beauchampia, avec comme type Beauchampia alluaudi.

Selon Yamaguti, ce genre se caractérise par: un ovaire situé en arrière du niveau des vagins, mais antérieur, un utérus postovarien occupant le champ intercaecal, des œufs embryonnés; un tube digestif avec anastomose haptoriale; un hapteur dépourvu d'hamulis.

L'examen du matériel de de Beauchamp nous a montré que cette interprétation de Yamaguti comporte une méprise fondamentale.

En effet, le schéma de de Beauchamp peut laisser croire qu'il existe un ovaire au niveau du quart antérieur de l'animal, bien que la description n'en fasse aucune mention; Yamaguti a adopté cette manière de voir. Or le détail en cause du dessin de 

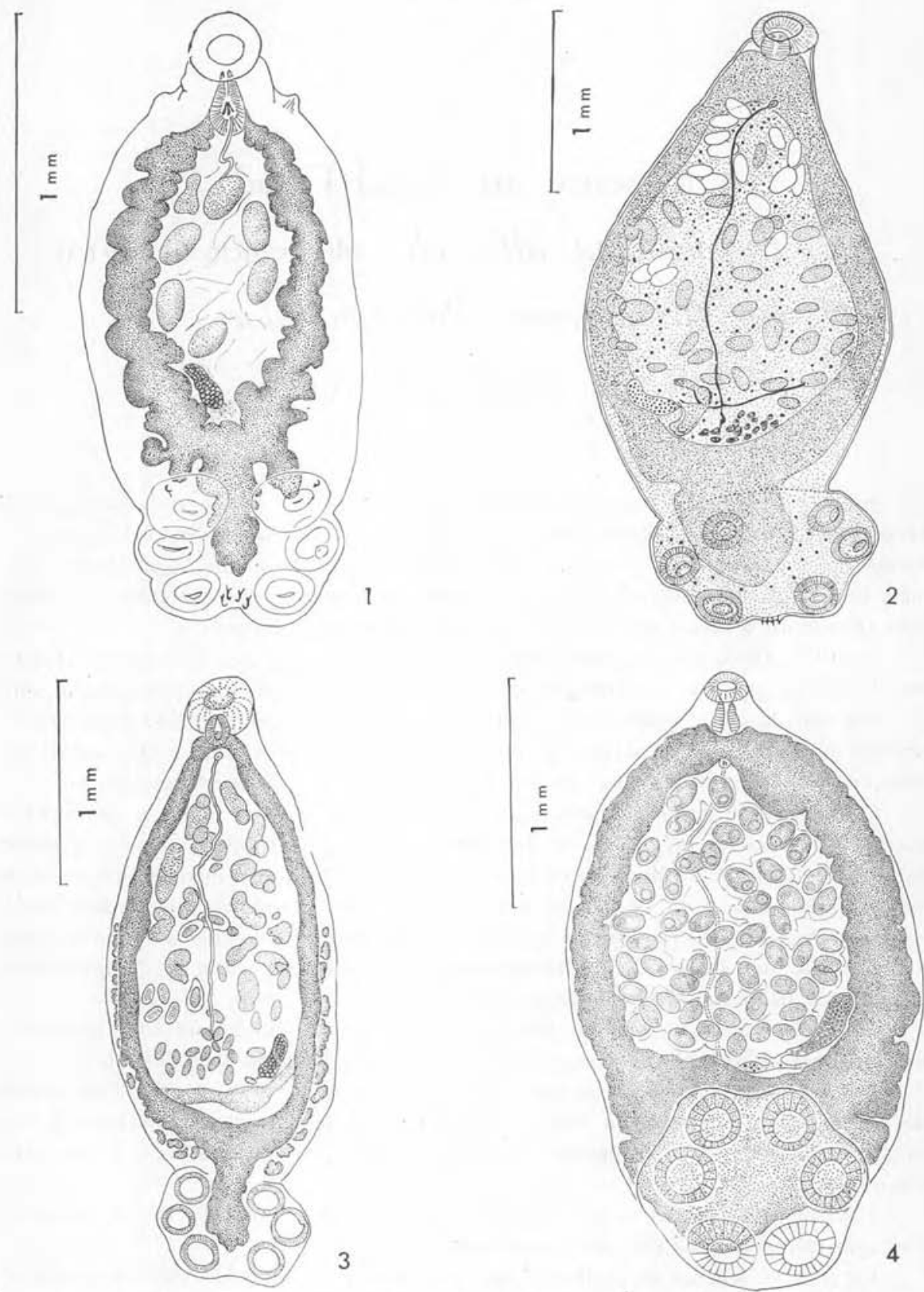

Fig. 1. - Eupolystoma alluaudi, d'après de Beauchamp, complété (ovaire) après examen de la préparation originale.

FIG. 2. - Eupolystoma rajai, d'après Kaw.

FIG. 3. - Eupolystoma alluaudi, d'après M. Beverley-Burton.

Fig. 4. - Eupolystoma alluaudi, exemplaire récolté au Nord-Tchad. . 
de Beauchamp ne représente qu'un œuf écrasé et déformé. L'ovaire authentique est situé dans la partie postérieure du corps, juste en avant de l'anastornose haptoriale (fig. 1).

A la suite de cette précision, l'animal devient alors tout à fait semblable à Eupolystoma rajai Kaw, 1950 (fig. 2). On peut même se demander si l'espèce de de Beauchamp et celle de Kaw ne sont pas synonymes; n'ayant pas à notre disposition le matériel de Kaw, nous pouvons seulement affirmer que ces deux espèces font partie du même genre Eupolystoma.

Nous donnons côte à côte la figure de Kaw pour E. rajai et notre dessin de l'exemplaire original décrit par de Beauchamp. On se rend compte de l'identité de leurs caractères essentiels. Nous donnons également copie du dessin de Beverley-Burton qui concerne manifestement lui aussi Eupolystoma alluaudi, et chez qui la ressemblance est encore plus frappante (fig. 3).

Au cours d'une Mission Epidémiologique au Nord-Tchad (1960), J.-A. Rioux a récolté chez un Amphibien non identifié (vraisemblablement un Bufo) un Polystomatidae qui présente tous les caractères de Eupolystoma alluaudi, ce qui constitue la troisième découverte de ce parasite (fig. 4).

Le biotope particulier (gorge de Beskéré) où l'Amphibien a été récolté, est schématisé par J.-A. Rioux dans le compte rendu de la mission (fig. 26, p. 81). Il s'agit d'une guelta isolée dans le massif désertique de l'Ennedi. Cela pose le problème de la répartition géographique de Eupolystoma alluaudi d'une part, le problème de son cycle biologique en fonction des mœurs de son hôte et du biotope dans lequel il évolue, d'autre part. Nous pensons que la présence, chez tous les Eupolystoma étudiés jusqu'à présent, d'œufs contenant une larve complètement formée, est à elle seule l'indice d'un cycle différent de celui des autres Polystomatidae.

Rappelons enfin que Reichenbach-Klinke a décrit sous le nom de Eupolystoma australensis un Monogène qui vit sur les branchies et la peau du Dipneuste Neoceratodus forsteri. Ce parasite, mis à part l'absence d'hamuli, ne présente aucun des caractères définis par Kaw pour le genre Eupolystoma. Par contre, il nous paraît très voisin de Pseudopolystoma dentriticum (Ozaki 1948), parasite d'un Urodèle. En particulier l'absence d'hamuli et de vagin, la position antérieure de l'ovaire, le testicule divisé en cystes, l'utérus renfermant un seul œuf, permettent ce rapprochement. La seule différence consiste dans la présence d'une anastomose digestive haptoriale chez l'espèce de Reichenbach-Klinke, anastomose qui fait défaut chez l'espèce d'Ozaki. Le parasite de Neoceratodus forsteri doit prendre le nom de Pseudopolystoma australensis (Reichenbach-Klinke 1966).

\section{Bibliographie}

Beauchamp (P.-M. de), 1913. - Polystomum alluaudi n. sp. Voyages de Ch. Alluaud et R. Jeannel en Afrique Orientale (1911-1912). Résultats scientifiques, Turbellariés, Trématodes et Gordiacés, p. 17-19, fig. 1. 
Beverley-Burton (M.), 1962. - Some monogenetic trematodes from Amphibia in Southern Rhodesia including a new species, Polystoma mashoni, sp. n., from Bufo regularis (Reuss). Journ. Parasitol., 48 (5), p. 752-757, 2 pl.

Kaw (B. L.), 1950. - Studies in Helminthology: Helminth parasites of Kashmir. Part I. Trematoda. Ind. J. Helm., 2 (2), p. 67-126.

OzaKI (Y.), 1935. - Studies on the Frog-Trematode Diplorchis ranae. I. Morphology of the Adult Form with a Review of the Family Polystomatidae. Journ. Sc. Hirosh. Univ., Ser. B, Div. 1, 3 (16), p. 193-223, fig. 1-28.

Reichenbach-KlinKe (H.), 1966. - Eine neue Art der Polystomatidengattung Eupolystoma Kaw 1950 (Monogenea, Polystomatidae) von der Kiemen des Australischen wagenfisches Neoceratodus forsteri Kreff. Zool. Anz. 176 (2), p. 142-146, fig. 1-5.

Rioux (J. A.), 1960. - Misison Epidémiologique au Nord-Tchad. Comité de coordination scientifique du Sahara, Paris, p. 1-132, fig. 1-29.

Yamaguti (S.), 1963. - Systema Helminthum. Vol. IV. Monogenea. Int. Publ. New York, p. $1-699,134 \mathrm{pl}$.

(Laboratoire de Zoologie et Biologie animale du Collège Scientifique Universitaire 66 - Perpignan) 